\title{
Surveillance System and Prevalence of Healthcare-Associated Infections in a Maternity Hospital
}

\author{
Pagona Flevari, ${ }^{1}$ Irene Zorou, ${ }^{2}$ Athanassios Tsakris, ${ }^{1}$ and George Saroglou ${ }^{2}$ \\ ${ }^{1}$ Department of Microbiology, Medical School, University of Athens, 11527 Athens, Greece \\ ${ }^{2}$ Internal Medicine Clinic, "Elena Venizelou” Maternity Hospital, Nursing School, University of Athens, 11521 Athens, Greece \\ Correspondence should be addressed to Athanassios Tsakris; atsakris@med.uoa.gr
}

Received 17 June 2013; Accepted 5 August 2013

Academic Editors: J. Bustamante and R. Favory

Copyright (c) 2013 Pagona Flevari et al. This is an open access article distributed under the Creative Commons Attribution License, which permits unrestricted use, distribution, and reproduction in any medium, provided the original work is properly cited.

\begin{abstract}
Obstetrician-gynaecologist (Ob-Gyn) medical and nursing personnel usually have minimal in action training on infection control (IC). Limited information exists also about the epidemiology of healthcare-associated infections (HAIs) in maternity hospitals. The aim of this study was to determine in a 30-day survey prevalence of HAIs among hospitalised patients and neonates in a maternity hospital in Athens, Greece, and to offer to the IC office a practical IC manual. Patients hospitalized for more than $24 \mathrm{~h}$ in the clinics and the neonatal intensive care unit (NICU) were enrolled. An IC guide was created and distributed to the medical and nursing staff through educational seminars. Through the survey, among Ob-Gyn patients 16 HAIs were recognized during hospitalization and 14 HAIs after patients' discharge; the overall prevalence of infected patients was $2.9 \%$ and the prevalence of HAIs was $3.2 \%$. Among NICU patients, the prevalence of HAIs was 3.9\%. The IC manual was found easily implemented in daily use improving staff's compliance to IC practices. The results of the survey can be used as a baseline for future comparisons between maternity hospitals (benchmarking). The implementation of steady IC guideline protocols for a maternity hospital may update staff education and promote staff compliance on IC practices.
\end{abstract}

\section{Introduction}

The impact of healthcare-associated infections (HAIs) is significant, since not only do they adversely affect patient outcome by increasing morbidity and mortality, but also they prolong hospital stay, enhance resistance of microorganisms to antibiotics, and add economic burden [1-3]. An overview of published reports has suggested that at least $20 \%$ of all nosocomial infections can be avoidable, depending on the setting, study design, baseline infection rates, and type of infection [4], while another literature review has pointed out that surveillance of HAIs as part of the IC program can reduce infection rates by up to $30 \%$ or more [5]. However, the epidemiology of HAIs in maternity hospitals, which are characterized by a special patient population, has not yet been well defined.

In many maternity hospitals there are gaps regarding the implementation of IC practices and the staff orientation and training. Obstetrician-Gynaecologist (Ob-Gyn) patients have a short length of hospital stay and a significant number of
HAIs are detected after hospital discharge. Typically, in a survey for the epidemiology of postpartum infections the overall infection rate was $6 \%$ with rates of $7.4 \%$ following caesarean section and 5.5\% following vaginal delivery; however, most of them were registered after hospital discharge [6]. Moreover, critically ill neonates in intensive care units have immature immune system, underlying health problems, and undergo invasive diagnostic and therapeutic procedures, characteristics that give them a high-risk profile for developing HAIs. In national point prevalence surveys almost onefifth of neonatal intensive care unit (NICU) patients have been reported to suffer from nosocomial infections, infants with low birth weight were more likely to have an infection, and therapeutic intervention was significantly associated to NICU-acquired infections $[7,8]$.

The impact of HAIs not only is of increasing concern, but also underlines clearly the significance of a well-organized IC program. Maternity hospitals should mandate the presence of an IC committee to oversee and coordinate IC practices through dissemination of information, surveillance activities, 
investigation, antibiotic stewardship, prevention, and control of HAIs. The aim of the present study, conducted in a maternity hospital, was to measure the infection rates as well as to develop and implement a proper IC manual, based on the hospital's needs, function, and resources. The manual including methods, such as educational seminars, was distributed to the personnel to enhance the staff compliance to IC practices.

\section{Materials and Methods}

2.1. Clinical Setting. The study was conducted at "Elena Venizelou" Maternity Hospital, a 314-bed maternity hospital with $97 \%$ occupancy located in Athens, Greece. It provides medical care for Athens and the surrounding area (approx. a population of 5,000,000). A 30-day period prevalence survey of HAIs was conducted. All patients hospitalized for more than $24 \mathrm{~h}$ in the Ob-Gyn clinics and NICU were enrolled.

2.2. Definitions. HAIs were defined according to Centres for Disease Control and Prevention's National Nosocomial Infection Surveillance system definitions [9-11]. In case of neonates, symptoms and positive cultures within $48 \mathrm{~h}$ after birth were considered as infections transmitted vertically from mother to newborn or perinatal infections. Infections manifested $48 \mathrm{~h}$ or more after admission in the NICU were defined as healthcare associated.

2.3. Methodology of Nosocomial Infection Surveillance. Files and charts collecting data about patient's sex, age, medical history, present diagnosis, medication, intervention procedures, and exposure to invasive devices, surgery, clinical course, fever, and duration of hospitalization were reviewed. The clinical laboratory provided information about identification of bacterial pathogens and their antimicrobial susceptibility profiles. Findings from the radiology examinations (X-rays, Ultrasounds, and CTs) were also evaluated. All hospitalised patients developing infection were examined. All Ob-Gyn patients enrolled in the study were contacted by phone 15 days after their hospital discharge and data indicating infection, such as fever or the consumption of antibiotics, were collected. All the above data were registered at questionnaires, for adult patients and neonates.

The prevalence of nosocomial infections (defined as the number of HAIs divided by the total number of patients hospitalized at the time of the study) and the prevalence of infected patients (defined as the number of infected patients divided by the total number of patients hospitalized at the time of the study) were calculated.

\subsection{Observation and Infection Control Manual Development} and Distribution. After daily observation of the medical and nursing staff routine by the IC nurse an IC manual with guidelines was created and distributed to the personnel, through educational seminars dealing with the implementation of practices described in the IC manual. The observation was then continued in order to evaluate the guide's practical use and the staff compliance to it. The chapters included in the IC manual and the content of each chapter are presented below.

Titles of Chapters and Their Content of the Infection Control (IC) Manual for Maternity Hospital.

Hand hygiene: with pictures showing the technique of handwashing with soap and water or with alcohol-based hand rub and with pictures showing the surgical hand preparation.

Medical gloves, scrubs and masks: when and how to use them.

Preparing the woman before caesarean section: vaginal preparation with povidone-iodine solution and skin preparation with povidone-iodine scrub, and paint immediately before caesarean delivery.

Medical sharps and waste: how to handle and to dispose them to the special containers.

Methods of decontamination of instruments and the environment: disinfection and sterilization in order to prevent cross-infection from equipment, surfaces and skin.

Infections that after exposal to them a healthcare worker must receive chemoprophylaxis: hepatitis $\mathrm{A}$, hepatitis $\mathrm{B}$, rabies, whooping cough, diphtheria, and meningococcal disease.

Management of exposure to HIV, HBV, and HCV: serological tests and chemoprophylaxis.

Immunization practices of healthcare workers.

Care of intravascular devices and catheter insertion: hand washing, barrier precautions, technique, catheter site care, antisepsis, dressing regimens, monitoring, replacement frequency, and indications.

Isolation: which patients need isolation, ward's equipment, patient's care, and immunocompromised patient.

The manual included the necessary course of action regarding the hospital's IC guidelines about hand hygiene, medical gloves, scrubs and masks, medical sharps and waste, decontamination of instruments and the environment, infections that after exposal to them a health-care worker must receive chemoprophylaxis, management of exposure to HIV, HBV, HCV, immunization practices, care of intravascular devices, and catheter insertion and patients' isolation policy.

\section{Results and Discussion}

3.1. Prevalence of HAIs in the Ob-Gyn Clinics. During the surveillance period a total of 1,030 women were hospitalized in the Ob-Gyn clinics. Sixteen of them developed HAIs during their hospitalization, while 14 developed HAIs after their hospital discharge; in the latter cases HAIs were recognised by phone contact. The mean hospitalization time was 3 days. Among the infected patients, 27 (90\%) developed a single HAI, while $3(10 \%)$ developed two HAIs. The overall prevalence of infected patients was $2.9 \%$ and the prevalence of HAIs was $3.2 \%$. There were 7 patients with surgical site infections, 4 patients with urinary tract infection $(2$ of them had also infection of the lower respiratory system), 3 patients each with gastroenteritis and mastitis ( 1 of the latter patients had also infection of the lower respiratory system), one patient with chorioamnionitis, thrombophlebitis, and uterine infection, and 10 patients with fever of unknown origin (treated 
TABLE 1: Type of infections among Ob-Gyn patients developing HAIs in maternity clinics during the 30 -day period prevalence survey.

\begin{tabular}{lcc}
\hline Type of infection & $\begin{array}{c}\text { Number of } \\
\text { infected patients }\end{array}$ & $\begin{array}{c}\text { Percentage of } \\
\text { infected patients }\end{array}$ \\
\hline Fever of unknown origin & 10 & $34 \%$ \\
Surgical site infection & 7 & $23 \%$ \\
Gastroenteritis & 3 & $10 \%$ \\
Urinary tract infection & 2 & $7 \%$ \\
Urinary tract infection and & 2 & $7 \%$ \\
lower respiratory system & & \\
infection & 2 & $7 \%$ \\
Mastitis & 1 & $3 \%$ \\
Mastitis and lower respiratory & 1 & $3 \%$ \\
system infection & 1 & $3 \%$ \\
Uterine infection & 1 & $3 \%$ \\
Chorioamnionitis & & \\
Thrombophlebitis & & \\
\hline
\end{tabular}

TABLE 2: Prevalence of neonates with HAIs in the ICU during the 30day period prevalence survey.

\begin{tabular}{lcc}
\hline Type of infection & $\begin{array}{c}\text { Number of } \\
\text { infected neonates }\end{array}$ & $\begin{array}{c}\text { Percentage of } \\
\text { infected neonates }\end{array}$ \\
\hline Fever of unknown origin & 2 & $29 \%$ \\
Eyes infection & 2 & $29 \%$ \\
$\begin{array}{l}\text { Skin and soft tissue } \\
\text { infection }\end{array}$ & 2 & $28 \%$ \\
Bloodstream infection & 1 & $14 \%$ \\
\hline
\end{tabular}

with wide spectrum antibiotics) (Table 1). As many as 24 of the 30 patients (80\%) with HAIs underwent caesarean section (note that a total of 346 caesarean sections were performed during the surveillance period), indicating that caesarean section is associated with high morbidity for HAI [12]. In our survey, the high prevalence of HAIs among women who underwent caesarean section could be also attributed to lack of proper infection control measures.

3.2. Prevalence of HAIs in the NICU. During the study period 176 neonates were hospitalized in the NICU and 7 of them developed HAIs. All infected neonates developed a single HAI. Thus, the prevalence of infected neonates as well as the prevalence of HAIs was 3.9\%. There were two neonates with skin infection, two with eye infection, one with bloodstream infection due to MRSA (positive blood culture), while two had experienced fever of unknown origin (Table 2). There was no neonate mortality during the study period.

3.3. Detailed Creation of an IC Guide and Education of the Hospital Personnel. The observation of the staff daily routine was initially conducted by the IC nurse. It was realized that when the IC nurse appeared on the ward, the staff acted appropriately (due to the awareness of being studied), but the nurse's presence was soon to be forgotten. Despite the plethora of evidence-based guidelines, policies and procedures available nowadays on IC, suboptimum care was given because knowledge and understanding the importance of the above guidelines were still limited. The introduction of the IC manual through the educational seminars had considerable consequences in hospital's IC practices. The medical and nursing staff found the IC manual easy to implement in daily use, and through further observation the personnel's compliance was considerably promoted and improved. The IC manual was found a tool assisting the user to create safe practices as well as to realize that patients' care will benefit from enhancing IC standards.

3.4. Discussion. The present 30-day prevalence survey was conducted in a hospital setting with patients' characteristics substantially different from those in other institutions and clinics. The specific population under evaluation was characterized by short hospital stays (Ob-Gyn patients) and high risk for acquisition of HAIs (NICU patients with immature immune system and underlying health problems as mentioned above). It is also of note that in the current survey the same observer team and standardized methodology were used. The study provided the baseline of our hospital's infection rates as well as the essential observations in order to suggest a proper and feasible surveillance program.

The review of updated patients' medical records is an essential step for recognizing changes in the clinical course during their hospitalization. In particular, the sensitivity and specificity of this method in measuring nosocomial infection rates has been well defined in the literature [13]. Accurate record keeping should be prioritized as part of a purposefully designed monitoring system that upgrades IC. It have been emphasized that healthcare facilities even in countries with limited resources should participate actively to the organization of a hospital's electronic data base $[14,15]$.

During surveillance of HAIs a lot of data need to be collected for every patient, such as demographic and clinical data, admission date, medical history, primary diagnosis, date of recognizing the infection, and the type of infection. The IC personnel must be able to define in the best possible way the population at high risk and, in the case of an epidemic, the exact number of infected patients, the place, and the time the outbreak has initiated. During the present surveillance the probability of a hidden epidemic should be excluded. Situations that may cause artefacts in the estimation of HAIs prevalence rates such as modified definition of an infection, implementation of the surveillance program to a new department or population, new laboratory methods, or changes in medical treatments, and interventional procedures were not applied to our hospital setting.

The prevalence of nosocomial infections is underestimated in some cases especially when wide spectrum antibiotics are administered without defining the type of the infection. Quick response to antibiotics and elimination of the infection signs may result the nonrecognition of many HAIs. In our study, as many as $34 \%$ of our Ob-Gyn patients with fever remained undiagnosed receiving widespectrum antibiotics, without their doctors implementing 
first a protocol of investigating the fever. Since the initiation of our study we have changed this obviously not proper "policy" through antibiotic stewardship.

Decreased hospitalization time in maternity clinics leads to an increased rate of HAIs that appear after patient's discharge [16]. An effective postdischarge programme increases significantly the nosocomial infection rates and can include methods such as contacting the patient by mail or phone, sending questionnaires to the medical personnel, and screening of electronic records, 15 days after hospital discharge $[17,18]$. Although this overloads those who are responsible for conducting it, this procedure ensures larger amount of data, accuracy, and reliability on infection rates. Our study showed that nearly $50 \%$ of the obstetric HAIs manifested after hospital discharge.

Understaffing and low levels of staff preparedness and knowledge are key factors leading to insufficient IC practices. Implementation of IC standards is difficult in the case of poor hygiene and sanitation, equipment shortage, inadequate structures, overcrowding, and limited surveillance data, such as about antibiotic consumption and HAIs epidemiology [4]. Therefore education, recruitment of IC professionals, and distribution to the hospital staff of an IC manual with simple and applicable prevention measures and tools are essential particularly for highly demanding units, such as those of maternity hospitals.

\section{Conclusion}

In conclusion, the standards for proper IC include the strategic development of a healthcare-associated infections surveillance system, infection control education, and antibiotic stewardship. The present survey provided essential data and observations in order to suggest a surveillance program not only for our maternity hospital but also for all such facilities in order to reduce infection rates $[4,19]$. An antibiotic stewardship program with antibiotic consumption measurement was also initiated in our hospital [20]. This would help to design interventions that will ensure that hospitalized patients receive the proper antimicrobial treatment reducing thus antimicrobial resistance.

IC is no longer to be regarded as low priority but as a costeffective measure, and all efforts must be directed towards achieving attitude changes in healthcare personnel. It is feasible to make a real change, introducing to the staff a manual with practical tools that include operation, information, education, monitoring, hand hygiene, product procurement, and impact evaluation. An updated IC manual and an established educational program through seminars by the IC team seem to promote awareness of IC procedures and behavioural and organizational changes. Surveillance, quality improvement measures, organisational and behavioural changes, continuous education, antibiotic stewardship, and globalized health policies and initiatives are considered essential in order to reduce maternal infection rates.

\section{Conflict of Interests}

The authors declare that there is no conflict of interests.

\section{References}

[1] P. W. Stone, E. Larson, and L. N. Kawar, "A systematic audit of economic evidence linking nosocomial infections and infection control interventions: 1990-2000," American Journal of Infection Control, vol. 30, no. 3, pp. 145-152, 2002.

[2] K. B. Laupland, H. Lee, D. B. Gregson, and B. J. Manns, "Cost of intensive care unit-acquired bloodstream infections," Journal of Hospital Infection, vol. 63, no. 2, pp. 124-132, 2006.

[3] S. K. Pada, Y. Ding, M. L. Ling et al., "Economic and clinical impact of nosocomial meticillin-resistant Staphylococcus aureus infections in Singapore: a matched case-control study," Journal of Hospital Infection, vol. 78, no. 1, pp. 36-40, 2011.

[4] S. Harbarth, H. Sax, and P. Gastmeier, "The preventable proportion of nosocomial infections: an overview of published reports," Journal of Hospital Infection, vol. 54, no. 4, pp. 258-266, 2003.

[5] P. Gastmeier, "Nosocomial infection surveillance and control policies," Current Opinion in Infectious Diseases, vol. 17, no. 4, pp. 295-301, 2004.

[6] D. S. Yokoe, C. L. Christiansen, R. Johnson et al., "Epidemiology of and surveillance for postpartum infections," Emerging Infectious Diseases, vol. 7, no. 5, pp. 837-841, 2001.

[7] A. H. Sohn, D. O. Garrett, R. L. Sinkowitz-Cochran et al., "Prevalence of nosocomial infections in neonatal intensive care unit patients: results from the first national point-prevalence survey," Journal of Pediatrics, vol. 139, no. 6, pp. 821-827, 2001.

[8] E. Sarvikivi, T. Kärki, and O. Lyytikäinen, "Repeated prevalence surveys of healthcare-associated infections in Finnish neonatal intensive care units," Journal of Hospital Infection, vol. 76, no. 2, pp. 156-160, 2010.

[9] J. S. Garner, W. R. Jarvis, T. G. Emori, T. C. Horan, and J. M. Hughes, "CDC Defionitions for nosocomial infections, 1988," American Journal of Infection Control, vol. 16, no. 3, pp. 128-140, 1988.

[10] T. C. Horan, R. P. Gaynes, W. J. Martone, W. R. Jarvis, and T. G. Emori, "CDC definitions of nosocomial surgical site infections, 1992: a modification of CDC definitions of surgical wound infections," Infection Control and Hospital Epidemiology, vol. 13, no. 10, pp. 606-608, 1992.

[11] W. C. van der Zwet, A. M. Kaiser, R. M. van Elburg et al., "Nosocomial infections in a Dutch neonatal intensive care unit: surveillance study with definitions for infection specifically adapted for neonates," Journal of Hospital Infection, vol. 61, no. 4, pp. 300-311, 2005.

[12] V. P. Ward, A. Charlett, J. Fagan, and S. C. Crawshaw, "Enhanced surgical site infection surveillance following caesarean section: experience of a multicentre collaborative post-discharge system," Journal of Hospital Infection, vol. 70, no. 2, pp. 166-173, 2008.

[13] R. W. Haley, D. R. Schaberg, and D. K. McClish, "The accuracy of retrospective chart review in measuring nosocomial infection rates. Results of validation studies in pilot hospitals," American Journal of Epidemiology, vol. 111, no. 5, pp. 516-533, 1980.

[14] R. A. Leth and J. K. Møller, "Surveillance of hospital-acquired infections based on electronic hospital registries," Journal of Hospital Infection, vol. 62, no. 1, pp. 71-79, 2006.

[15] R. A. Leth, M. Nørgaard, N. Uldbjerg, R. W. Thomsen, and J. K. Møller, "Surveillance of selected post-caesarean infections 
based on electronic registries: validation study including postdischarge infections," Journal of Hospital Infection, vol. 75, no. 3 , pp. 200-204, 2010.

[16] R. C. Couto, T. M. G. Pedrosa, J. M. Nogueira, D. L. Gomes, M. F. Neto, and N. A. Rezende, "Post-discharge surveillance and infection rates in obstetric patients," International Journal of Gynecology and Obstetrics, vol. 61, no. 3, pp. 227-231, 1998.

[17] J. McNeish, D. Lyle, M. McCowan, S. Emmerson, S. McAuley, and J. Reilly, "Post-discharge surgical site infection surveillance by automated telephony," Journal of Hospital Infection, vol. 66, no. 3, pp. 232-236, 2007.

[18] E. S. Petherick, J. E. Dalton, P. J. Moore, and N. Cullum, "Methods for identifying surgical wound infection after discharge from hospital: a systematic review," BMC Infectious Diseases, vol. 6, article 170, 2006.

[19] R. W. Haley, "The scientific basis for using surveillance and risk factor data to reduce nosocomial infection rates," Journal of Hospital Infection, vol. 30, supplement, pp. 3-14, 1995.

[20] P. Flevari, V. Bentouli, E. Kada et al., "Three-year antibiotic stewardship in a Greek maternity hospital," Archives of Hellenic Medicine, vol. 27, no. 2, pp. 208-214, 2010. 


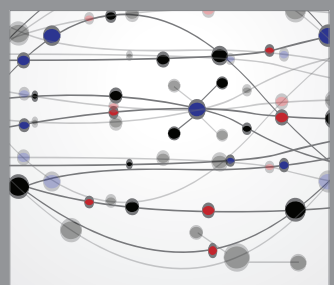

The Scientific World Journal
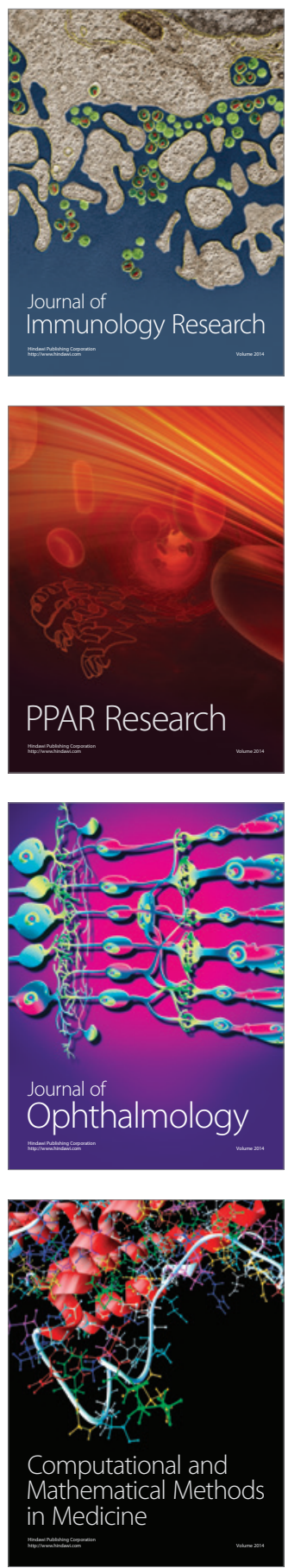

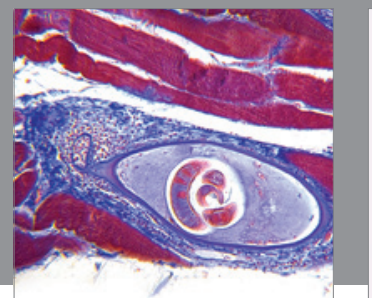

Gastroenterology

Research and Practice
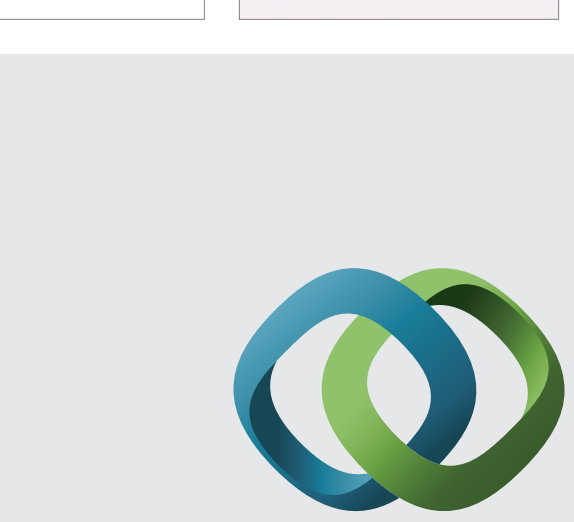

\section{Hindawi}

Submit your manuscripts at

http://www.hindawi.com
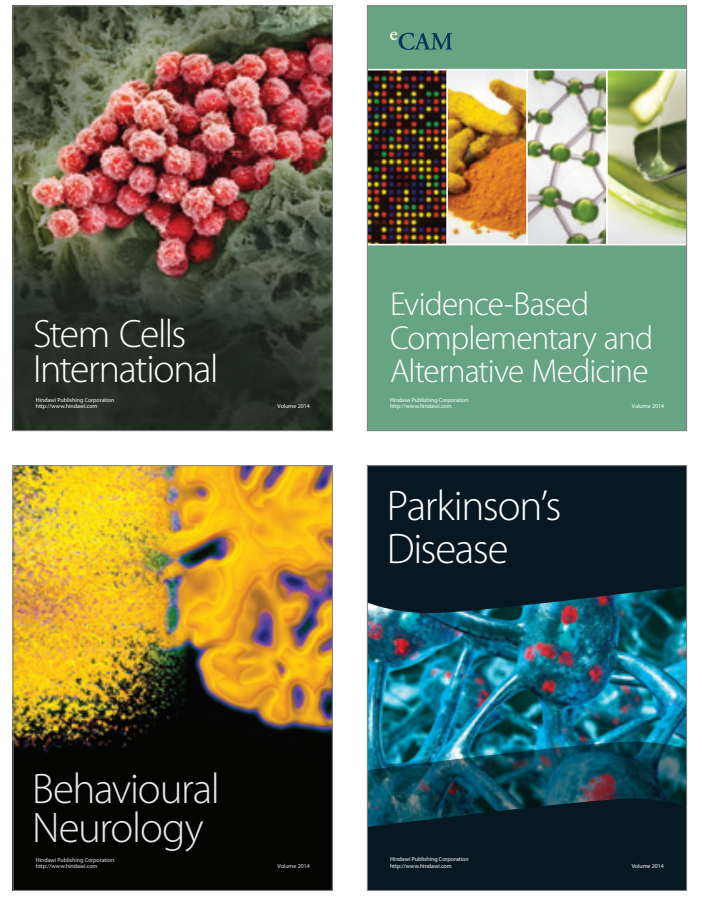
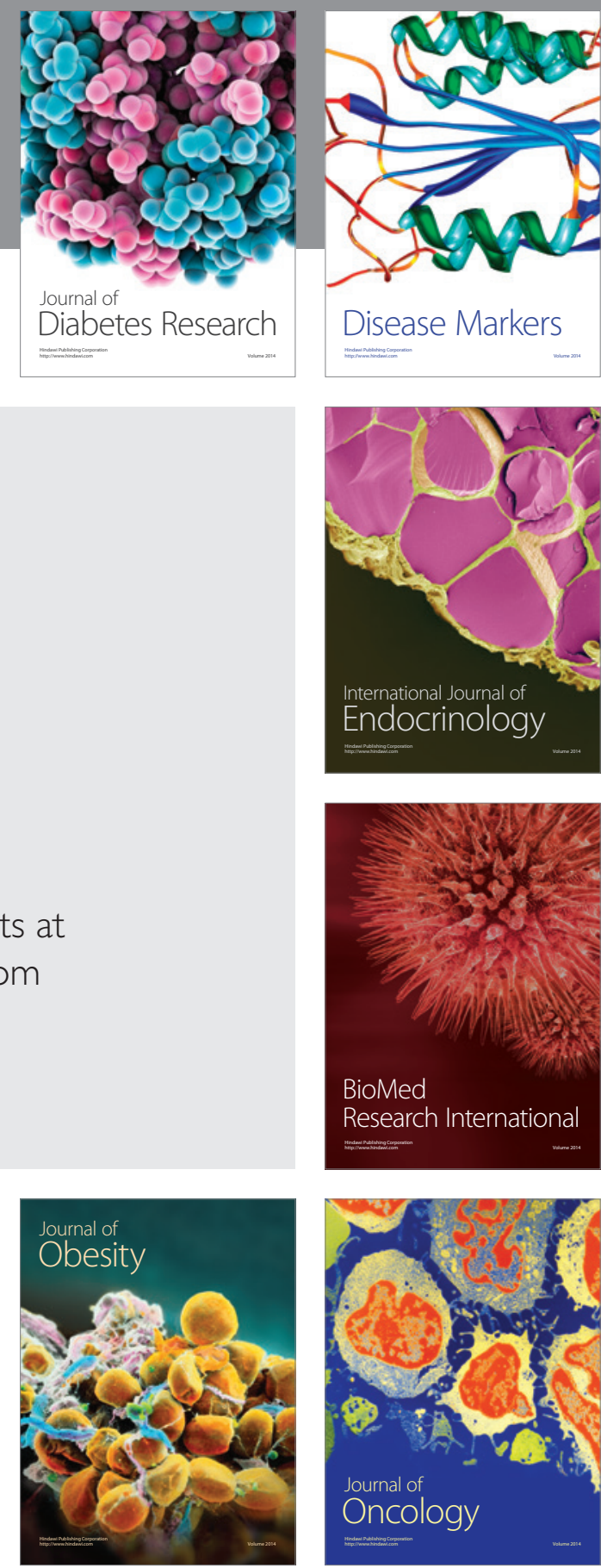

Disease Markers
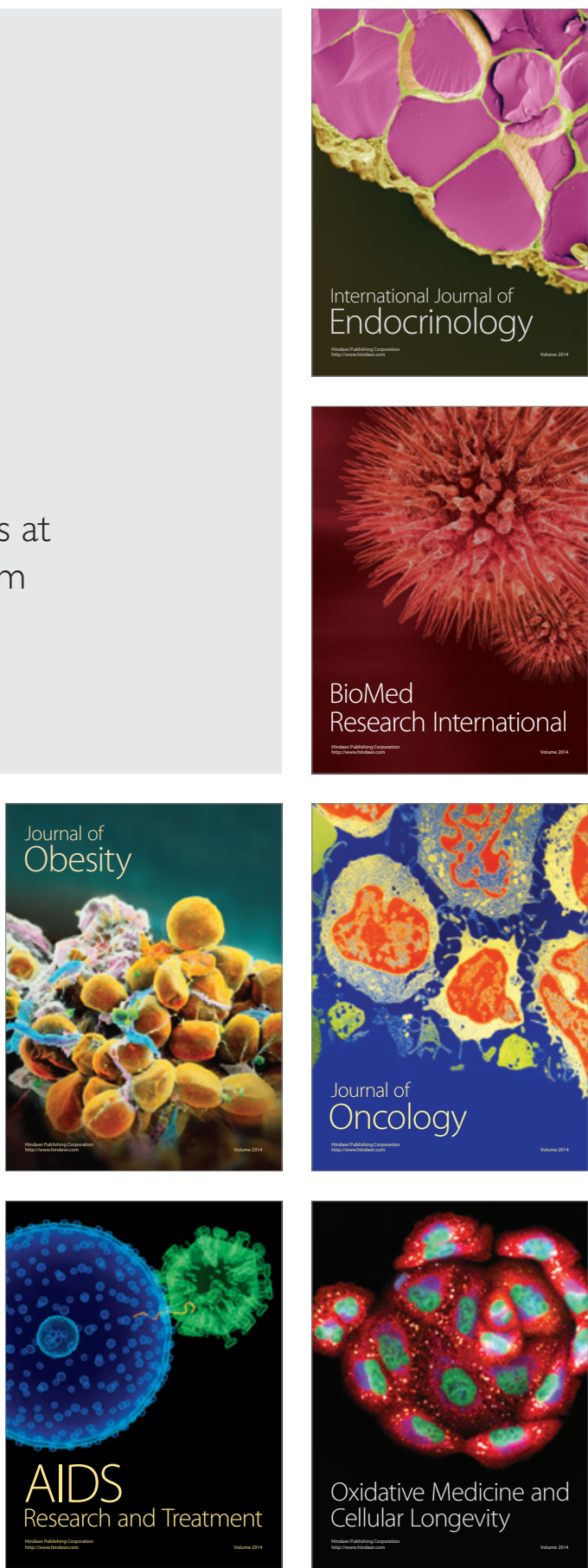